\title{
An Enhanced Automated Diagnosis Method for Glaucoma Detection Using Wavelet
}

\author{
${ }^{1}$ D. Evangelin Hepsiba, ${ }^{2}$ A. Josephine Puspha Arasi
}

\begin{abstract}
Glaucoma is a disease of the eye, characterized by increased Intraocular Pressure (IOP), excavation and atrophy of optic nerve. Without treatment, glaucoma can cause total permanent blindness. A technique for automated screening of glaucoma from retinal fundus images using wavelet features is presented in this paper. The discussed wavelet features such as mean and entropy values are obtained by using daubechies (db3) wavelet filter. Thus the features are validated by classifying the normal and glaucoma images using Artificial Neural Network (ANN). The performance is analysed with evaluation metrics in terms of accuracy and specificity. Thus the observed accuracy and specificity is $79 \%$ and $100 \%$ respectively.

Keywords: Glaucoma, fundus, wavelet feature, daubechies (db3), Artificial Neural Network (ANN).
\end{abstract}

\section{Introduction}

Worldwide, Glaucoma is the second leading cause of blindness after cataract [1]. It affects one in two hundred people aged 50 \& younger and one over the age of 80. In India, people at age limit of 40 are affected by glaucoma and the estimate is around 11.2 million [2].

Glaucoma is a term describing a group of ocular disorder with multi-factorial etiology united by a clinically characteristic Intraocular Pressure (IOP) associated optic neuropathy. The nerve damage involves loss of retinal ganglion cells in a characteristic pattern. Raised IOP (above $21 \mathrm{mmHg}$ ) is the most important and risk factor for glaucoma.

Untreated glaucoma can lead to permanent damage of optic nerve and resultant visual loss over time can progress to blindness [4]. Till now there is no cure for glaucoma so an early diagnosis and preventive measure is to be carried to avoid vision loss [1].

The disease can be classified into two major types. It includes the open angle glaucoma and angle closure glaucoma. In case of open angle glaucoma the eye appears to be normal. The fluid does not properly flow through the drain of the eye. This type accounts for $90 \%$ of glaucoma cases. Eye vision is affected gradually. If detected earlier it can be treated by meditation. Angle-closure glaucoma is also called as acute or chronic angle-closure or narrow-angle glaucoma, this type of glaucoma is less common but can cause a sudden build up of pressure in the eye.

The presence of glaucoma can be identified by certain test such as ophthalmoscopy, tonometry and perimetry. The detection test consumes more time. Nowadays, computer based techniques plays a vital role in the glaucoma diagnoses. Thus OCT and HRT techniques are widely used for diagnosing [1]. Even though these techniques are available the cost of machinery is too high and hence it will not be available in all the hospitals $[5]$.

\section{Materials Used}

The retinal images used for this study are collected from Rimone database. Around 160 images are used. All the images are taken at a resolution of 512*512 pixels and stored in BMP format. The images that are collected are separated into four categories such as normal and glaucoma eye images.

The glaucoma affected eye images are further grouped into three and taken as Early, Moderate and Deep. Fig.1(a) and (b) shows a typical normal and glaucoma fundus image respectively.

\section{Methodology}

The objective of image processing system is to extract the relevant features for the automatic diagnosis of the glaucoma. Here in our experimentation the desired features such as mean and entropy of the images are calculated using wavelet transform.

A. Wavelet Based Segmentation

In any image processing system, the first and foremost step is pre-processing followed by segmentation. The input images are pre-processed. 


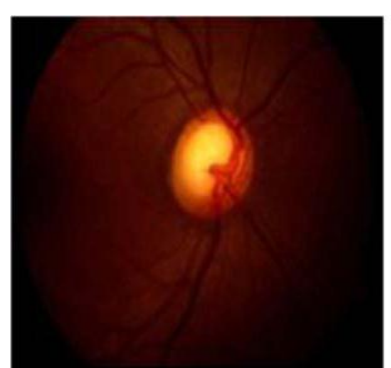

(a)

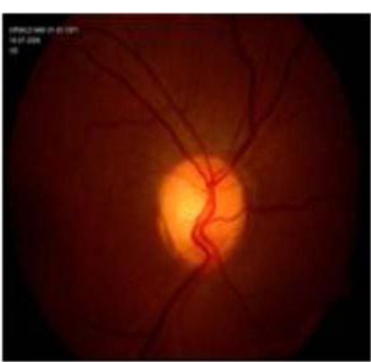

(b)

Fig.1 (a) Normal image (b) Glaucoma image

Segmentation is carried out by means of wavelet transform. It decomposes the input images into approximate and detailed coefficients [4]. Then the detailed coefficients are further decomposed into three such as horizontal, vertical and diagonal components. Here we are considering three levels of decomposition so totally 84 features are obtained.

The segmentation of the optic disc is carried out by active contour method [6] pixel classification and contour modelling [7] threshold techniques. Meanwhile detection of optic disc can be carried out by matched filter [8] also.

\section{B. Feature Extraction}

Wavelet Packet Transformation is an extension of wavelet decomposition [9]. It is mainly used in image/signal processing applications. Thus in our study, the feature extraction is carried out by the wavelet function daubechies (db3) wavelet filter.

The obtained mean and entropy features are summed up and taken as significant features. The mean feature matrix and the entropy feature matrix values are calculated. The matrix consists of the image and feature value represented by rows and columns respectively.

\section{Final Classification}

An Artificial Neural Network (ANN) is an information processing unit that is inspired by the way biological nervous systems processes information. The network is composed of a large number of highly interconnected neurons working together to solve specific tasks [1].

Neural networks are simplified models that resemble human brain. It acquires knowledge through learning and the inter neuron connection is called synaptic weights that store knowledge. Data enters at the inputs and passes through the layer of hidden layers, where the actual information is processed and the result is available at the output layer. Back propagation architecture is used for our experimentation.

Initially the system weights are randomly chosen and then slowly modified during the training to in order to get the desired outputs. The difference between the actual output and desired output is calculated for each input at each iteration. These errors are used to change the weights proportionately. This algorithm of reducing the errors in order to achieve the correct class by incrementing weights is known as back propagation [1].

The three layer network will be able to solve most of the complex problems with sigmoid activation function. The non-linearity of the sigmoid function makes the back propagation learning algorithm powerful in classification process. The output neuron will classify the images as ' 1 ' for normal and '2' for glaucoma. The classification is carried out by two phase the training and testing phase.

From the dataset 128 images are used for training and 32 images for testing. The network was trained with given set of training data and later tested with remaining testing samples. .In the training phase, the mean and entropy performance are calculated. Then the images are randomized in order to avoid successive selection of images. Then in the testing phase, the actual and the target values of the corresponding images are compared and the performance metrics are calculated.

\section{Results And Discussion}

The results of our experiments are represented by means of performance metrics such as accuracy and specificity. The output values are represented in terms of bar chart shown in Fig.2. 


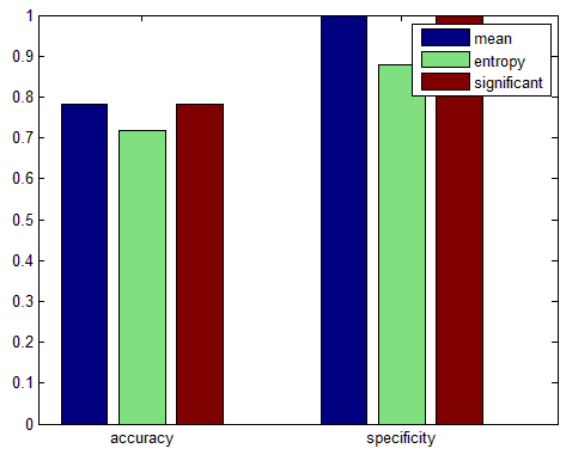

Fig.2 Bar chart of performance metrics

Thus the discussed wavelet features mean, entropy and significant feature values are obtained and represented graphically. The achieved accuracy and sensitivity is $79 \%$ and $100 \%$ respectively.

\section{Conclusion}

This paper provides an alternate approach by using wavelet. The presence of glaucoma can be identified by preferring this method. Thus the features are identified and corresponding accuracy and sensitivity is calculated.

\section{References}

[1] Jagadish Nayak, Rajendra Acharya U,P. Subbanna Bhat,Nakul Shetty,Teik-Cheng Lim, "Automated Diagnosis of Glaucoma Using Digital Fundus Images, "Journal of Medical Systems, Vol.33,no.5,October 2009

[2] R. George, R. S. Ve, and L. Vijaya, "Glaucoma in India: Estimated burden of disease,” J. Glaucoma, vol. 19, pp. 391-397, Aug. 2010 .

[3] J. M. Miquel-Jimenez et al., "Glaucoma detection by wavelet-based analysis of the global flash multifocal electroretinogram," Med. Eng. Phys., vol. 32, pp. 617-622, 2010.

[4] Sumeet Dua, Senior Member, IEEE, U. Rajendra Acharya, Pradeep Chowriappa, Member, IEEE, and S. Vinitha Sree, "WaveletBased Energy Features for Glaucomatous Image Classification" IEEE transcations on information technology in biomedicine,Vol. 16, No. 1, January 2012.

[5] 'Optic Disk and Cup Segmentation from Monocular Color Retinal Images for Glaucoma Assessment', Gopal Datt Joshi, Member, IEEE, Jayanthi Sivaswamy, Member, IEEE, and S. R. Krishnadas'” IEEE transcation on medical imaging, December, 2010.

[6] 'Automatic Localization and Boundary Detection of Optic Disc Using Implicit Active Contours', Siddalingaswamy P. C, Gopalakrishna Prabhu .K, 2010 International Journal of Computer Applications (0975 - 8887) Volume 1 - No. 7.

[7] 'Automated segmentation of optic disc region on retinal fundus photographs: Comparison of contour modeling and pixel classification methods', Chisako Muramatsu, Toshiaki Nakagawa, Akira Sawada, Yuji Hatanaka, Takeshi Hara, Tetsuya Yamamoto, Hiroshi Fujita, Journal of computer methods and programs in biomedicine 101 ( 2011 ) 23-32.

[8] 'Optic Disc Detection From Normalized Digital Fundus Images by Means of a Vessels Direction Matched Filter' by Aliaa AbdelHaleim Abdel-Razik Youssif, Atef Zaki Ghalwash, Amr Ahmed Sabry Abdel-Rahman Ghoneim. IEEE transcation on medical imaging, VOL. 27, NO. 1, January 2008.

D.EVANGELIN HEPSIBA, from Trichy completed Bachelors degree in Electronics and Communication Engineering, from J.J. College Of Engineering and Technology, Anna University, Chennai in the year 2012. Completed Master degree in Computer and Communication Engineering, from M.A.M College Of Engineering, Anna University, Chennai in the year 2014.

A.JOSEPHINE PUSPHA ARASI, from Trichy, is the Associate Professor, Department of Information and Technology, M.A.M College Of Engineering, Siruganur, Trichy. 